\title{
Can the survival recall advantage be explained by basic memory processes?
}

\author{
YANA WEINSTEIN \\ University College London, London, England \\ AND \\ Julie M. Bugg And Henry L. Roediger III \\ Washington University, St. Louis, Missouri
}

\begin{abstract}
Nairne, Thompson, and Pandeirada (2007) demonstrated a striking phenomenon: Words rated for relevance to a grasslands survival scenario were remembered better than identical words encoded under other deep processing conditions. Having replicated this effect using a novel set of words (Experiment 1), we contrasted the schematic processing and evolutionary accounts of the recall advantage (Experiment 2). Inconsistent with the schematic processing account, the grasslands survival scenario produced better recall than did a city survival scenario requiring comparable schematic processing. Recall in the grasslands scenario was unaffected by a self-reference manipulation. The findings are consistent with an evolutionary account that attributes the recall advantage to adaptive memory biases.
\end{abstract}

Evolutionary psychologists (e.g., Tooby \& Cosmides, 1992, 2005) propose that our cognitive processes have been shaped by adaptation. One premise is that given the ultimate goal of staying alive and reproducing, humans may have evolved to heighten attention in situations where their survival is threatened or simply made salient. Following this reasoning, one might further predict that humans would exhibit enhanced retention for information that is processed while they are thinking about survival, or when they are in a survival-mode. In order to test this prediction, Nairne, Thompson, and Pandeirada (2007) asked participants to imagine a scenario in which they would have to secure food and shelter and protect themselves from predators, and then rate words for their relevance to the scenario. This encoding task led to higher levels of recall than did control conditions in which the same words were rated for relevance to moving to a foreign land, pleasantness, or self-relevance, and the latter two tasks have been shown in prior research to be among the best encoding tasks for enhancing later recall and recognition, as discussed below.

Nairne, Thompson, and Pandeirada's (2007) findings are remarkable for several reasons. First, the findings stem from one of the few paradigms that have been established to empirically test an evolutionary-based explanation of memory performance. Second, the findings provide, at least at first glance, some rather convincing evidence in favor of the idea that memory for information processed while one is in a survival mode is superior to memory for information that is deeply processed by virtue of other en- coding manipulations. Since Craik and Lockhart's (1972) influential article, differences in free recall rates following various encoding manipulations have typically been attributed to depth of processing during encoding. That is, semantically processed information is generally recalled at a higher rate than is information processed for shallow attributes, such as phonemic, visual, or auditory properties (but see Roediger \& Gallo, 2002, for some difficulties with this account). Though there are various means by which one can deeply encode information, Packman and Battig (1978) showed that rating words for pleasantness resulted in significantly higher recall than that obtained in six other tasks, including rating words for meaningfulness. Subsequently, Challis, Velichkovsky, and Craik (1996) showed that rating words for relevance to oneself produced higher rates of free recall than did a variety of other semantic rating tasks. As such, pleasantness and self-reference rating tasks have generally been perceived as the most conducive encoding tasks studied in this tradition; it is therefore noteworthy that Nairne, Thompson, and Pandeirada have revealed the existence of an encoding task that results in higher levels of free recall than those found with other tried and true methods.

Because research in this area is just beginning, it is not entirely clear whether the survival advantage is reliable or extends to other materials. At the outset of our project, no reports of replication in independent laboratories or with a different set of stimuli had appeared. To preface, in Experiment 1 , we first established that the survival advantage is indeed replicable and generalizes to a new set of words.

Y.Weinstein, y.weinstein@ucl.ac.uk 
Experiment 2 was designed to test a more theoretically interesting question: By what process is memory performance facilitated when participants evaluate the relevance of words to a survival scenario? Nairne, Thompson, and Pandeirada's (2007) view is that the advantage reflects a deeply rooted adaptive bias that is activated when participants are asked to read the survival scenario and evaluate words on its basis. Another view is that the survival processing advantage reflects the operation of basic memory processes such as enhanced schematic or self-referential processing, not abstract, evolutionary biases. In Experiment 2, we contrasted these competing accounts.

\section{EXPERIMENT 1}

Participants rated lists of 12 words in three different encoding conditions and later performed a surprise free recall test. Two of the encoding conditions involved rating words for their relevance to a particular scenario (survival or moving), whereas the other condition involved rating words for pleasantness. The encoding and recall tasks were similar in nature to those used in Nairne, Thompson, and Pandeirada (2007); no changes were made to the wording of the orienting tasks, although we used a completely within-subjects design incorporating all three rating scenarios and a new set of word stimuli. Nairne, Thompson, and Pandeirada had not included all three orienting tasks in a within-subjects design, so this experiment represents a modest extension as well as replication of prior work.

\section{Method}

\section{Participants}

Seventy-two paid participants took part in this experiment, of whom 36 were undergraduate students from Washington University in St. Louis and 36 were volunteers recruited at University College London.

\section{Materials and Design}

Stimuli were 36 concrete nouns randomly selected from a list of words that were generated by submitting a range of 400-700 for the frequency, concreteness, imageability, and meaningfulness criteria in the MRC Psycholinguistic Database (see the Appendix). This list was then randomly split into three lists of 12 words. As can be seen, we made no special effort to select words for survival relevance. A within-subjects design was used, with rating scenario (survival/ moving/pleasantness) as the independent variable. All participants studied the same three lists of 12 words, with the order of lists, order of rating scenario, and assignment of word lists to scenario counterbalanced, though the order of words within each list was held constant. Altogether, this produced 36 counterbalancing conditions, and 2 participants were assigned to each of these conditions.

\section{Procedure}

Participants were informed that they would be rating words on the basis of various characteristics. They were then given one of the following three rating instructions, which were identical in wording to those used in Nairne, Thompson, and Pandeirada's (2007) experiments.

Survival. "In this task we would like you to imagine that you are stranded in the grasslands of a foreign land, without any basic survival materials. Over the next few months, you'll need to find steady supplies of food and water and protect yourself from predators. We are going to show you a list of words, and we would like you to rate how relevant each of these words would be for you in this survival situation. Some of the words may be relevant and others may notit's up to you to decide."

Moving. "In this task we would like you to imagine that you are planning to move to a new home in a foreign land. Over the next few months, you'll need to locate and purchase a new home and transport your belongings. We are going to show you a list of words, and we would like you to rate how relevant each of these words would be for you in accomplishing this task. Some of the words may be relevant and others may not-it's up to you to decide."

Pleasantness. "In this task, we are going to show you a list of words, and we would like you to rate the pleasantness of each word. Some of the words may be pleasant and others may not-it's up to you to decide."

On each trial of the rating task, a word appeared in the center of the screen, and participants were asked to rate it using a 5-point scale where $1=$ totally irrelevant or totally unpleasant and $5=$ extremely relevant or extremely pleasant. The rating scale appeared on the screen below each presented item, and participants indicated their response by selecting the appropriate key on the number pad. Critically, participants were unaware that memory for the items that they rated would subsequently be tested. They were asked to respond within $5 \mathrm{sec}$ and reaction times (RTs) were recorded. If a response was not made within $5 \mathrm{sec}$, the next trial started automatically and data from the trial did not contribute to RT and ratings means presented below. Such trials were extremely rare and resulted in only 10 missing values across both experiments.

After participants rated 12 words in one scenario, they received instructions for the next rating task, until all three scenarios had been completed. Following completion, participants performed a 2-min filler task in which they were asked to recall as many U.S. states as possible (Washington University participants) or calculate math sums (University College London participants). After the filler task, participants were asked to write down as many words as they could remember from the rating tasks, in any order. Ten minutes were given for this recall task.

\section{Results and Discussion}

The primary purpose of Experiment 1 was to examine the replicability of the survival advantage in free recall obtained by Nairne, Thompson, and Pandeirada (2007). Levels of free recall achieved in each of the three encoding conditions are shown in Figure 1. Consistent with their findings, the survival advantage was obtained using a within-subjects design. That is, a significant main effect of rating scenario was found for free recall $[F(2,142)=$ $\left.3.62, M S_{\mathrm{e}}=223, p<.05\right] .{ }^{1}$ Recall was $5.2 \%$ greater for words rated in terms of their relevance to survival as compared with their relevance to moving $[t(71)=2.27$, $S E M=2.30, p<.05]$ and $6.3 \%$ higher than for words rated for pleasantness $[t(71)=2.37, S E M=2.63, p<$ $.05]$, as indicated by planned paired-samples $t$ tests. This pattern of findings represents a conceptual replication of the work of Nairne, Thompson, and Pandeirada and extends the survival advantage to a novel set of word stimuli, obviating concern that the effect might be bound to a specific set of stimuli.

To confirm that the recall pattern that we obtained was not driven by differences between the conditions in either RTs to produce ratings or mean ratings, we conducted within-subjects ANOVAs on the mean RT and ratings data, which are shown in Table $1 .{ }^{2}$ Rating scenario had a significant effect on RTs $\left[F(2,136)=6.58, M S_{\mathrm{e}}=80,725, p<\right.$ $.01]$; post hoc comparisons indicated that the times taken to rate items for relevance to survival and moving did not 


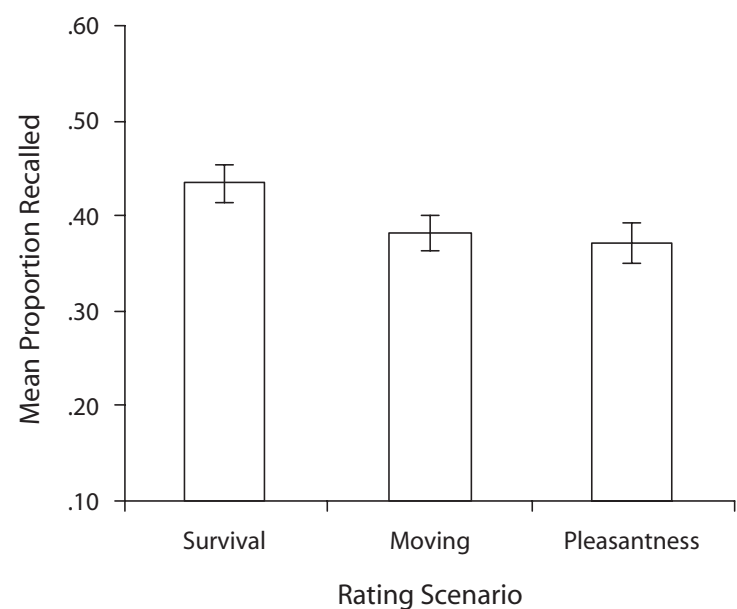

Figure 1. Mean proportion of words recalled by rating scenario in Experiment 1. The error bars represent standard errors of the means.

differ $(p=.44)$, whereas pleasantness ratings were made significantly faster than survival ratings $[t(68)=2.85$, $S E M=45, p<.01]$. Although one might argue that the additional time spent producing the ratings in the survival condition relative to the pleasantness condition might account for the survival recall advantage over pleasantness, we believe that a simple processing time explanation of this sort is unlikely. Such an explanation would also predict equivalent recall levels for the survival and moving conditions, because the time taken to produce ratings in the survival and moving conditions was equivalent. As confirmed earlier, this was not the case. In addition, prior research in the levels-of-processing tradition has shown that type of task is critically important in producing differences in recall, and time spent on the encoding task has negligible effects (Craik \& Tulving, 1975; Hyde \& Jenkins, 1973).

Rating scenario also had a significant effect on mean ratings $\left[F(2,136)=33.07, M S_{\mathrm{e}}=0.17, p<.001\right]$. Although survival and moving ratings did not differ $(p=.49)$, words were rated as significantly more pleasant than relevant to survival $[t(68)=7.34, S E M=0.06, p<.001]$. Clearly, the latter two rating tasks cannot be treated as equivalent: In the case of the survival task, ratings are made in relation to a particular scenario, whereas pleasantness ratings are made by assigning valences. In both cases, however, we might expect that higher ratings would lead to higher levels of recall because of a congruity effect (Craik \& Tulving, 1975). Because the pattern of ratings that we found here would be expected to enhance, not attenuate, memory for

Table 1

Mean Ratings and Reaction Times (RTs, in Milliseconds) by Rating Scenario in Experiment 1

\begin{tabular}{lcccc}
\hline Rating Scenario & RT & $S E$ & Rating & $S E$ \\
\hline Survival & 1,618 & 49 & 2.62 & 0.06 \\
Moving & 1,656 & 57 & 2.50 & 0.07 \\
Pleasantness & 1,489 & 40 & 3.04 & 0.05 \\
\hline
\end{tabular}

words rated for pleasantness as opposed to survival, alternative explanations of the survival advantage based simply on scenario-word relevance may be discounted.

To summarize, Experiment 1 provided a conceptual replication of the results of Nairne, Thompson, and Pandeirada (2007), demonstrating a significant memory advantage for survival over moving and pleasantness encoding using a novel set of words and a completely withinsubjects design. Experiment 2 was conducted to evaluate the processes that underlie this memory advantage.

\section{EXPERIMENT 2}

One specific account of the recall advantage is that evaluating words for their relevance to the survival scenario encourages greater schematic processing than do conditions such as rating pleasantness, and this in turn facilitates memory performance (Bransford \& Johnson, 1972). A straightforward evaluation of the schematic processing account would pit the survival scenario against a second scenario that is presumed to encourage equally rich schematic processing. Nairne, Thompson, and Pandeirada (2007) attempted to do this by using a control task in which words were rated for their relevance to moving to a foreign land. The survival scenario produced superior retention when compared with the moving scenario, suggesting that the advantage is not simply the result of enhanced schematic processing. This evidence might be considered tentative, however, because it could be argued that the two scenarios were not fully equated for schematic processing. Whereas the survival scenario engages participants in a rather unusual context, likely forcing them to perform online schematic processing to assign the relevance of each item, the moving scenario is not as novel. Rather than engaging in schematic processing online, participants could rely on existing schemas and stored experiences involving moving to rate words for relevance to the moving scenario.

An alternative approach for evaluating a schematic processing account is to use two scenarios that involve almost identical wording, with one referencing a context that evolutionary theorists would anticipate as inducing the survival mode (surviving predators in the grasslands) and the other involving a context that would be less likely to induce this ancestral survival mode (surviving attackers in a city). This approach results in two similar scenarios that are more equally matched in the level of online schematic processing required. If the previously observed recall advantage simply reflects disproportionately greater engagement in schematic processing while studying the survival scenario relative to prior comparison conditions (e.g., moving), then the advantage should be negated when one compares the two present scenarios, which are closely matched in the level of schematic processing required. Indeed, because college students are more accustomed to living in cities than in grasslands, one might predict that they could use schemas to elaborate words and concepts more readily in this scenario than in the grasslands survival scenario. If so, one might even expect better recall in the city survival scenario than in the grasslands survival scenario. 
On the other hand, the evolutionary account generates an opposing prediction. This line of thinking proposes that our attitude toward survival has been shaped by evolution, and thus threats to survival that were endured by our ancestors are more salient to us than threats that have appeared relatively recently in our evolutionary history (Öhman \& Mineka, 2001). Early research into phobias suggested that humans are more likely to be phobic regarding predators and open spaces than guns and dangerous vehicles even though the latter are more realistic threats in the modern environment (Marks, 1969; Seligman, 1971; but see Fox, Griggs, \& Mouchlianitis, 2007, for recent data on a competing account). The prediction that follows from this reasoning is that certain survival scenarios should activate the adaptive bias more strongly than others. Accordingly, in the present experiment, the evolutionary account anticipates recall to be greater when participants are encouraged to think about survival in the grasslands rather than survival in a more modern context such as a city.

A secondary goal of Experiment 2 was to evaluate another explanation of the recall advantage that, like the schematic processing account, is rooted in existing memory principles. This account attributes the recall advantage to greater engagement in self-referential processing in the survival scenario than in control conditions. One way in which self-referential processing is thought to affect memory is that it facilitates high levels of elaboration (Rogers, Kuiper, \& Kirker, 1977). The idea is that when relating a concept to ourselves, we are able to engage in more item-specific processing because we are so proficient at thinking about the self (Kihlstrom, 1993). If the survival advantage is partially dependent upon engagement in self-referential processing, one might hypothesize that it should weaken if participants are asked to consider the relevance of words to someone else's survival instead of their own. To evaluate this prediction, we also manipulated perspective in Experiment 2 in such a way that instructions were either phrased in the 1 st person (imagine yourself in this scenario) or in the 3rd person (imagine $a$ friend in this scenario).

\section{Method}

\section{Participants}

Eighty-eight participants were drawn from the University College London participants' pool and either were paid the equivalent of $\$ 4$ for their time or volunteered their time without monetary reward. Paid/unpaid participants were split evenly among all conditions.

\section{Materials and Design}

We manipulated both survival context (city, grasslands) and perspective (1st person, 3rd person) between subjects: Participants rated words for relevance either to survival in the grasslands $(n=$ $40)$ or to survival in the city $(n=48)$; and half of each of these groups imagined themselves in this survival scenario, whereas the other half imagined a friend in the scenario. All participants also rated words for relevance to a moving scenario as an additional control to establish the basic survival advantage. Moving instructions remained the same regardless of survival scenario, but varied as to whether participants imagined themselves or a friend to match the survival scenario. We used the same 36 words from Experiment 1 and distributed them into four blocks of 9 words. The words were rated in the same order by all participants. Participants performed each of the two rating tasks (survival, moving) twice, and counterbalancing was achieved by rotating the order of scenarios across two conditions (moving, survival, moving, survival and survival, moving, survival, moving).

\section{Procedure}

The overall procedure and instructions were identical to those of Experiment 1, except that participants received new rating instructions after every nine words. The 1 st person moving and 1st person grasslands survival scenarios were identical to those used by Nairne, Thompson, and Pandeirada (2007) and by us in Experiment 1. The rest of the instructions were created by changing as few words as possible in order to retain the grammatical and syntactical structure of the text. Instructions for all the conditions are presented below. Note that italics are used here purely to draw attention to the differences between rating scenarios and were not presented in the instructions that the participants received.

Grasslands survival (1st person). "In this task we would like you to imagine that you are stranded in the grasslands of a foreign land, without any basic survival materials. Over the next few months, you'll need to find steady supplies of food and water and protect yourself from predators. We are going to show you a list of words, and we would like you to rate how relevant each of these words would be for you in this survival situation. Some of the words may be relevant and others may not-it's up to you to decide."

Grasslands survival (3rd person). "In this task we would like you to imagine that a friend is stranded in the grasslands of a foreign land, without any basic survival materials. Over the next few months, they'll need to find steady supplies of food and water and protect themselves from predators. We are going to show you a list of words, and we would like you to rate how relevant each of these words would be for them in this survival situation. Some of the words may be relevant and others may not-it's up to you to decide."

City survival (1st person). "In this task we would like you to imagine that you are stranded in the city of a foreign land, without any basic survival materials. Over the next few months, you'll need to find steady supplies of food and water and protect yourself from attackers. We are going to show you a list of words, and we would like you to rate how relevant each of these words would be for you in this survival situation. Some of the words may be relevant and others may not-it's up to you to decide."

City survival (3rd person). "In this task we would like you to imagine that a friend is stranded in the city of a foreign land, without any basic survival materials. Over the next few months, they'll need to find steady supplies of food and water and protect themselves from attackers. We are going to show you a list of words, and we would like you to rate how relevant each of these words would be for them in this survival situation. Some of the words may be relevant and others may not-it's up to you to decide."

Moving (1st person). "In this task we would like you to imagine that you are planning to move to a new home in a foreign land. Over the next few months, you'll need to locate and purchase a new home and transport your belongings. We are going to show you a list of words, and we would like you to rate how relevant each of these words would be for you in accomplishing this task. Some of the words may be relevant and others may not-it's up to you to decide."

Moving (3rd person). "In this task we would like you to imagine that a friend is planning to move to a new home in a foreign land. Over the next few months, they'll need to locate and purchase a new home and transport their belongings. We are going to show you a list of words, and we would like you to rate how relevant each of these words would be for them in accomplishing this task. Some of the words may be relevant and others may not-it's up to you to decide."

After the recall task, participants completed a Shipley vocabulary test (not analyzed here) and a demographic questionnaire. They were also asked to indicate the frequency with which they watched survival-related television programs (e.g., Survivor, Lost) using the following scale: never, sometimes, frequently, always. 


\section{Results and Discussion}

We first compared mean recall for the three instructional conditions. The grasslands survival condition produced highest recall (.38), the city survival condition produced next best recall (.31), and the moving condition produced the least recall (.28). We confirmed that an overall survival advantage (mean of grasslands and city survival) over moving was again obtained: Across all 88 participants, recall was on average $6.7 \%$ higher for words that were rated for relevance to any survival scenario than for words that were rated for relevance to the moving scenario $\left[F(1,87)=15.38, M S_{\mathrm{e}}=128, p<.001\right]$. Of the 88 participants, 53 showed this advantage, while 21 showed the opposite pattern; an exact binomial sign test confirmed that these proportions were significantly different $(p<$ .001). Furthermore, the survival advantage remained significant in the 42 participants who reported never watching survival-related television programs $[F(1,41)=7.30$, $\left.M S_{\mathrm{e}}=158, p<.05\right]$. The overall finding of an advantage of survival over moving conceptually replicates that of Experiment 1; rates of recall in the moving condition were excluded from all further analyses. As discussed below, the grasslands survival scenario produced better recall than did the city survival scenario.

Of primary interest in Experiment 2 were two theoretically motivated questions. The first considered the degree to which disproportionate schematic processing accounts for the survival recall advantage; the second considered the role of self-referential processing in the effect. To preface, the survival recall advantage persisted when the control condition was matched for the degree of schematic processing required. Furthermore, this advantage was generally similar for both the 1 st and 3rd person perspectives, disfavoring a pure self-referential processing account.

The recall data for the relevant conditions are shown in Figure 2. We carried out a $2 \times 2 \times 2$ ANOVA with survival context (city, grasslands), perspective (1st, 3rd person), and incentive (paid, unpaid) as between-subjects variables. ${ }^{3}$ This analysis revealed a main effect of survival context $\left[F(1,80)=9.72, M S_{\mathrm{e}}=162, p<.005\right]$ : Recall was $7.8 \%$ higher for words rated for relevance to grasslands survival than for words rated for relevance to city survival. Instructions that differed from the grasslands survival scenario only in terms of evolutionary relevance were significantly less conducive toward subsequent recall, disfavoring a schematic processing account.

As for the self-reference manipulation, no main effect of perspective on recall was found $(F<1)$, although there was a marginal interaction between survival context and perspective $\left[F(1,80)=3.12, M S_{\mathrm{e}}=162, p=.08\right]$. Our hypothesis regarding this manipulation was that if selfreferential processing is responsible for the survival advantage, there should be a reduction in recall when the instructions ask participants to imagine someone other than themselves in the survival scenario. Although this was indeed the case in the city survival condition, with participants who imagined themselves in this scenario recalling 3.0\% more words than did those who imagined a friend, participants who read the grasslands survival sce-

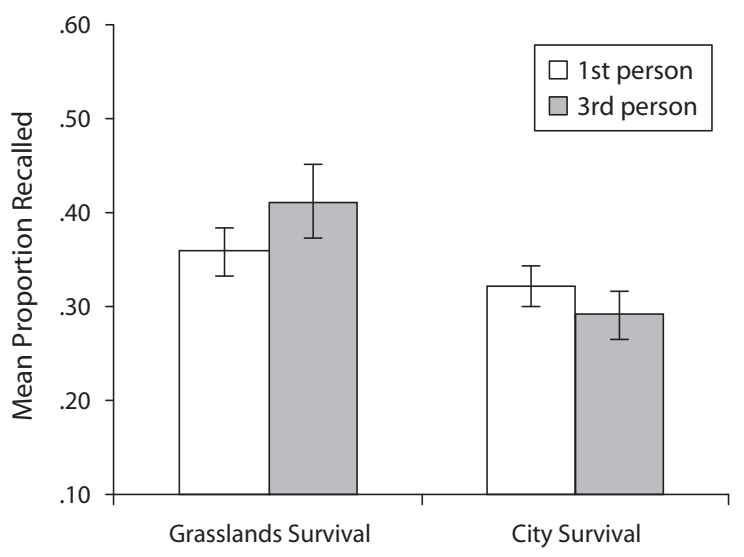

Encoding Condition

Figure 2. Mean proportion of words recalled by rating scenario in Experiment 2. The error bars represent standard errors of the means.

nario in the 3rd person actually performed $5.3 \%$ better than those who read the same scenario in the 1 st person. Neither of these comparisons approached significance ( $p$ s $>$.25). Obviously, the pattern of data showing, if anything, greater recall in the 3 rd person than in the 1 st person grasslands survival scenario cannot be accommodated by the notion that the basic survival advantage is created solely by self-referential processing.

In order to verify that the obtained pattern of recall was not dependent on ratings or RTs, ${ }^{4}$ we performed ANOVAs on the mean RT and ratings data with survival context (city, grasslands) and perspective (1st, 3rd person) as betweensubjects variables. The data are shown in Table 2. This analysis yielded no significant comparisons (all $p \mathrm{~s}>.14$ ): Scenario instructions did not affect mean ratings or time spent making these ratings, suggesting that the different conditions were indeed comparable.

In summary, Experiment 2 demonstrated that the recall advantage originally obtained by Nairne, Thompson, and Pandeirada (2007) persists even when the control condition is closely matched for schematic processing. This finding is particularly striking because the difference between encoding instructions was limited to two words, with city replacing grasslands and attackers replacing predators. This recall pattern is anticipated by the evolutionary account of the survival advantage and not the schematic processing account. As previously explicated, the evolutionary account

Table 2

Mean Ratings and Reaction Times (RTs, in Milliseconds) by Rating Scenario in Experiment 2

\begin{tabular}{lrrrr}
\hline \multicolumn{1}{c}{ Rating Scenario } & RT & $S E$ & Rating & $S E$ \\
\hline Survival: Grasslands $(n=40)$ & & & & \\
1st person & 1,761 & 111 & 2.51 & 0.14 \\
3rd person & 1,818 & 92 & 2.54 & 0.10 \\
Survival: City $(n=48)$ & & & & \\
$\quad$ 1st person & 1,640 & 108 & 2.49 & 0.14 \\
3rd person & 1,641 & 90 & 2.28 & 0.10 \\
\hline
\end{tabular}


predicts that survival contexts that would have been more relevant to our ancestors (e.g., grasslands) should activate the adaptive memory bias more strongly than those that would have been less relevant (e.g., modern city). Experiment 2 also demonstrated that the recall advantage was not limited to thinking about one's own survival. We further consider the implications of these findings for both the evolutionary and schematic processing accounts below.

\section{GENERAL DISCUSSION}

In two experiments, we have established three primary points. First, using a novel set of word stimuli, we have shown that processing words for relevance to survival results in better recall than either pleasantness ratings or processing words for relevance to a moving scenario. This finding confirms the survival recall advantage originally demonstrated by Nairne, Thompson, and Pandeirada (2007).

Second, we have shown that the recall advantage persists when the control condition is closely matched for the level of schematic processing required but differs from the original scenario in terms of evolutionary relevance. Specifically, rating words for relevance to survival in a modern, urban environment appears to be less conducive to recall than does rating words for relevance to survival in the grasslands. It is thus unlikely that the survival advantage can be explained in terms of the schematic processing account, which posits that the scenario instructions create a schema that is conducive toward elaboration and therefore leads to better encoding and retrieval. Instead, our finding is in line with an evolutionary account, according to which our memory has adapted very well to self-preservation in the type of setting in which we might have found ourselves until very recently (i.e., the grasslands), but has not yet evolved to function optimally in more modern contexts (i.e., the city). This is a reasonable prediction in light of the recent appearance of city-like urban settlements just over 5,000 years ago ("City," 2007), an insignificant event in terms of the larger evolutionary time scale of our development.

By pitting the grasslands survival scenario against an analogous scenario set in the city, we eliminated some of the apparent differences between the survival and moving scenarios. We more closely matched the two scenarios on at least four factors: the subject matter to which the scenarios referred; the grammatical structure and vocabulary of the scenario descriptions; the emotional arousal associated with the scenarios; and participants' first-hand experience with each scenario. In this way, the city scenario is an improvement over the original moving scenario. Nonetheless, we acknowledge that disagreement may remain regarding the equivalence of the city and grasslands survival scenarios. However, students in St. Louis and London are presumably more familiar with cities than with grasslands. If students applied and elaborated relevant schemas more readily in the former than in the latter scenario, one might have expected a city survival advantage to emerge rather than a grasslands survival advantage, as in other studies that have shown better retention for material when it can be encoded by readily accessible schemas (e.g., Bransford \& Johnson, 1972). This outcome clearly did not occur.

Although we have disconfirmed the schematic processing account, another account based on existing memory principles remains to be tested. The difference in recall between the grasslands and city survival conditions might be explained in terms of a proxy mechanism involving emotional arousal. It is well established that emotional arousal can lead to enhanced retention. The grasslands survival scenario may have evoked greater levels of emotional arousal, boosting recall relative to the potentially less arousing city survival scenario. Such an explanation is not, however, incompatible with an evolutionary account. It has been proposed, for example, that our fear module is responsive to evolutionarily primed stimuli (Öhman \& Mineka, 2001). Similarly, an evolutionary perspective posits that it would be adaptive to retain information about emotionally arousing stimuli in order to react to them more appropriately in the future (for a review, see Hamann, 2001). Further research is needed in order to tease apart these explanations.

The third finding that we established in the present study is that a self-reference manipulation (asking participants to imagine a friend instead of themselves in the survival scenario) had little effect on the levels of recall achieved. Similarly, Nairne, Thompson, and Pandeirada (2007, Experiment 4) found a significant memory advantage for words rated for relevance to survival in comparison with words rated for relevance to oneself. Taken together, the implication is that the survival advantage cannot be explained simply in terms of the degree to which the encoding task induces self-referential processing. Interestingly, while preparing this manuscript, we became aware of two additional pieces of research that converge with our own. Kang, McDermott, and Cohen (in press) have found that survival processing enhances retention even when words are rated for relevance to a character's survival in a video clip. Furthermore, Nairne, Pandeirada, and Thompson (2008) have shown that survival processing is better than a host of "deep" processing conditions, including selfreference. These data further strengthen the conclusion that a self-reference explanation of the survival advantage does not suffice.

One caveat of the finding above is that some research indicates that the effect of a self-reference manipulation may be related to the degree of intimacy of the subject in the 3rd person instructions (e.g., Bower \& Gilligan, 1979), although a meta-analysis found an overall significant effect of self-reference across 17 studies that used a highly intimate 3rd person (e.g., one's mother; Symons \& Johnson, 1997). In order to counter this concern, we ran an additional 18 participants in the 3rd person grasslands survival condition, with identical instructions except that a friend was replaced with a stranger. There was no difference in recall of words rated in the survival scenario between this condition and the analogous 1st person condition $(p=.76)$. Thus, the recall advantage does not appear to be limited to thinking about oneself in a survival scenario, but rather persists when words are processed for relevance to a friend or even a stranger. 
In summary, then, the adaptive survival advantage in free recall appears to be reliable and robust to changes in stimuli, rating tasks, and perspective. Critically, the recall advantage cannot be explained on account of at least two basic memory principles, schematic processing and self-reference. The primary finding, that recall is significantly higher when the instructions orient participants to an evolutionarily relevant (grasslands) as opposed to a modern (city) context accords nicely with an adaptive memory explanation of the sort posited by Nairne, Thompson, and Pandeirada (2007; see also Nairne \& Pandeirada, in press, for further discussion of this approach to the study of memory).

\section{AUTHOR NOTE}

This research was partially supported by BBSRC Studentship 07322/-101970 and a generous grant from the Bogue Fellowship, University College London. J.M.B. was supported by National Institute on Aging Grant 5T32AG00030 during the writing of this article. The authors thank James Nairne for comments on an earlier version of the manuscript. Correspondence concerning this article should be addressed to Y. Weinstein, Department of Psychology, University College London, Gower Street, London WC1E 6BT, England (e-mail: y.weinstein@ucl .ac.uk).

\section{REFERENCES}

Bower, G. H., \& Gilligan, S. G. (1979). Remembering information related to one's self. Journal of Research in Personality, 13, 420-432.

Bransford, J. D., \& Johnson, M. K. (1972). Contextual prerequisites for understanding: Some investigations of comprehension and recall. Journal of Verbal Learning \& Verbal Behavior, 11, 717-726.

Challis, B. H., VelichKovsky, B. M., \& CRaIK, F. I. M. (1996). Levelsof-processing effects on a variety of memory tasks: New findings and theoretical implications. Consciousness \& Cognition, 5, 142-164.

"Сiтү" (2007). In Encyclopcedia Britannica. Retrieved May 29, 2007, from Encyclopædia Britannica Online: www.britannica.com/eb/ article-9106247.

Craik, F. I. M., \& Lockhart, R. S. (1972). Levels of processing: A framework for memory research. Journal of Verbal Learning \& Verbal Behavior, 11, 671-684.

Craik, F. I. M., \& Tulving, E. (1975). Depth of processing and the retention of words in episodic memory. Journal of Experimental Psychology: General, 104, 268-294.

Fox, E., Griggs, L., \& Mouchlianitis, E. (2007). The detection of fear-relevant stimuli: Are guns noticed as quickly as snakes? Emotion, 7, 691-696.

HamanN, S. (2001). Cognitive and neural mechanisms of emotional memory. Trends in Cognitive Sciences, 5, 394-400.

HydE, T. S., \& JENKINS, J. J. (1973). Recall for words as a function of semantic, graphic, and syntactic orienting tasks. Journal of Verbal Learning \& Verbal Behavior, 12, 471-480.

KanG, S. H. K., McDermott, K. B., \& Cohen, S. M. (in press). The mnemonic advantage of processing fitness-relevant information. Memory \& Cognition.

Kinlstrom, J. F. (1993). What does the self look like? In T. K. Srull \& R. S. Wyer, Jr. (Eds.), The mental representation of trait and autobiographical knowledge about the self (pp. 79-90). Hillsdale, NJ: Erlbaum.

Marks, I. M. (1969). Fears and phobias. London: Heineman.

NaIRne, J. S., \& Pandeirada, J. N. S. (in press). Adaptive memory: Remembering with a stone-age brain. Current Directions in Psychological Science.

Nairne, J. S., Pandeirada, J. N. S., \& Thompson, S. R. (2008). Adaptive memory: The comparative value of survival processing. Psychological Science, 19, 176-180.

Nairne, J. S., Thompson, S. R., \& Pandeirada, J. N. S. (2007). Adaptive memory: Survival processing enhances retention. Journal of Experimental Psychology: Learning, Memory, \& Cognition, 33, 263-273.
Öhman, A., \& MineKa, S. (2001). Fears, phobias, and preparedness: Toward an evolved module of fear and fear learning. Psychological Review, 108, 483-522.

Packman, J. L., \& Battig, W. F. (1978). Effects of different kinds of semantic processing on memory for words. Memory \& Cognition, 6 , 502-508.

Roediger, H. L., III, \& Gallo, D. A. (2002). Levels of processing: Some unanswered questions. In M. Naveh-Benjamin, M. Moscovitch, $\&$ H. L. Roediger III (Eds.), Perspectives on human memory and cognitive aging: Essays in honour of Fergus Craik (pp. 28-47). New York: Psychology Press.

Rogers, T. B., Kuiper, N. A., \& Kirker, W. S. (1977). Self-reference and the encoding of personal information. Journal of Personality \& Social Psychology, 35, 677-688.

Seligman, M. E. P. (1971). Phobias and preparedness. Behavior Therapy, 2, 307-320.

Symons, C. S., \& Johnson, B. T. (1997). The self-reference effect in memory: A meta-analysis. Psychological Bulletin, 121, 371-394.

Tоову, J., \& Cosmides, L. (1992). The psychological foundations of culture. In J. H. Barkow, L. Cosmides, \& J. Tooby (Eds.), The adapted mind: Evolutionary psychology and the generation of culture (pp. 19-136). New York: Oxford University Press.

ToOby, J., \& Cosmides, L. (2005). Conceptual foundations of evolutionary psychology. In D. M. Buss (Ed.), The handbook of evolutionary psychology (pp. 5-67). Hoboken, NJ: Wiley.

\section{NOTES}

1. Note that for all analyses described below, a $3 \times 2$ mixed subjects ANOVA with rating scenario and sample (United Kingdom vs. United States) as factors was initially conducted for each of the three dependent measures. The main effect of sample was not significant for any measures, nor was the interaction of sample and rating scenario (all $p \mathrm{~s}>.1$ ). Therefore, we collapsed across the two samples for all reported analyses.

2. Because of a programming error, RT and ratings data are missing for 3 participants.

3. There was a significant main effect of incentive, showing that paid participants recalled $6.3 \%$ more words from the survival scenario than did unpaid volunteers $\left[F(1,80)=6.92, M S_{\odot}=5.46, p<.05\right]$. This factor did not interact with any other factors, and we therefore collapsed across this variable in subsequent analyses.

4. Ratings data were lost for 1 participant because of a programming error.

APPENDIX

\begin{tabular}{ll}
\multicolumn{2}{c}{ APPENDIX } \\
\hline AIR & MACARONI \\
AUTHOR & MAGAZINE \\
BACTERIA & OPIUM \\
BODY & PEACEMAKER \\
BOULDER & PIANO \\
BUNGALOW & PIPE \\
CANDY & PRIEST \\
CELLAR & PROPER'IY \\
CONTRACT & SHRIEK \\
CRADLE & SICKNESS \\
DOLL & SKIN \\
ENGINE & SLIPPER \\
GEM & SQUARE \\
GLUTTON & STRAWBERRY \\
GOLD & SUNSET \\
HOSTAGE & TIMEPIECE \\
INSTITUTE & TOMB \\
JOURNAL & WORLD \\
\hline
\end{tabular}

(Manuscript received November 26, 2007; revision accepted for publication January 22, 2008.) 